\title{
Do patients benefit from total intracorporeal robotic radical cystectomy?: A comparative analysis with extracorporeal robotic radical cystectomy from a Korean multicenter study
}

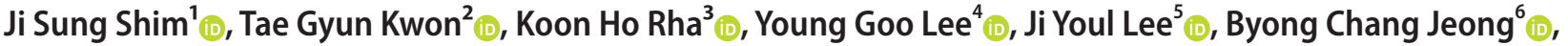 \\ Jong Hyun Pyun ${ }^{6}{ }_{(\mathbb{D})}$, Sung Gu Kang ${ }^{1}$ (D), Seok Ho Kang ${ }^{1}$ (i) \\ 'Department of Urology, Korea University College of Medicine, Seoul, ${ }^{2}$ Department of Urology, Kyungpook National University College of Medicine, Daegu, ${ }^{3}$ Department \\ of Urology, Yonsei University College of Medicine, Seoul, ${ }^{4}$ Department of Urology, Hallym University Kangnam Sacred Heart Hospital, Hallym University School of \\ Medicine, Seoul, ${ }^{5}$ Department of Urology, The Catholic University of Korea College of Medicine, Seoul, ${ }^{6}$ Department of Urology, Sungkyunkwan University College of \\ Medicine, Seoul, Korea
}

Purpose: This study aimed to compare complications, perioperative parameters, and oncologic outcomes between robot-assisted radical cystectomy (RARC) with extracorporeal urinary diversion (ECUD) and RARC with intracorporeal urinary diversion (ICUD).

Materials and Methods: Between 2007 and 2017, 362 patients who underwent RARC with ECUD or ICUD at multiple tertiary referral institutions were assessed. The primary endpoints were complication rates. The secondary outcomes were perioperative recovery parameters and oncological outcomes including estimated recurrence-free survival (RFS) and recurrence pattern between the 2 groups. Additionally, the complication rates of 2 expert surgeons with experience of $>100$ RARCs were analyzed.

Results: The ICUD group showed lower overall, gastrointestinal, and genitourinary complications $(p=0.001, p=0.036$, and $p=0.036$, respectively) than the ECUD group. Concerning perioperative outcomes, the ICUD group had a significantly longer operation time $(p=0.002)$, although recovery parameters such as time to flatus passage, oral intake, and length of hospital stay were significantly shorter in this group ( $p=0.001, p<0.001$, and $p<0.001$, respectively). There was no difference in oncologic outcomes such as positive margin rate $(p=0.944)$ and 2-year RFS $(p=0.496)$, and in the recurrence pattern between groups. In the comparison of the expert surgeons' complication rates, the major and total complication rates did not show differences $(p=0.814$ and $p=0.102$, respectively) while the minor complication rates were lower in the ICUD group ( $\mathrm{p}=0.058)$.

Conclusions: This multi-institutional cohort study demonstrated the benefits of the ICUD approach, as indicated by lower complication rates and better recovery parameters, although the oncological results were similar to those of ECUD.

Keywords: Cystectomy; Recurrence; Robotics; Urinary bladder neoplasms; Urinary diversion

This is an Open Access article distributed under the terms of the Creative Commons Attribution Non-Commercial License (http://creativecommons.org/licenses/by-nc/4.0) which permits unrestricted non-commercial use, distribution, and reproduction in any medium, provided the original work is properly cited.

Received: 29 March, 2019 - Accepted: 6 August, 2019

Corresponding Author: Seok Ho Kang (iD https://orcid.org/0000-0002-1524-5233

Department of Urology, Korea University College of Medicine, 73 Goryeodae-ro, Seongbuk-gu, Seoul 02841, Korea

TEL: +82-2-920-5530, FAX: +82-2-928-7864, E-mail: mdksh@korea.ac.kr 


\section{INTRODUCTION}

Since its adoption in 2003, robot-assisted radical cystectomy (RARC) has been steadily increasingly used worldwide, as a minimally invasive alternative to open radical cystectomy (RC) with comparable perioperative and oncologic outcomes [1,2]. Recently, the International Robotic Cystectomy Consortium collected 2,125 RARC cases and reported that the use of intracorporeal urinary diversion (ICUD) increased from $9 \%$ in 2005 to $97 \%$ in 2015 (rate of increase, $11 \%$ per year) [3].

Recent studies have suggested that RARC improves perioperative morbidity and facilitates recovery, which would theoretically allow for an earlier initiation of adjuvant systemic therapies $[4,5]$. These benefits result from the robot-assisted surgery itself, which is, by definition, a minimally invasive technique. Presently, clinicians have been debating whether urinary diversion (UD) during RARC is best completed extracorporeally or intracorporeally [6-9]. As most complications after RARC occur in association with UD, ICUD has been investigated as a way to reduce the rate of complications [10].

To maximize the advantages of minimally invasive sur- gery, a total ICUD approach may be a possible alternative. It is known that ICUD minimizes evaporative fluid loss; reduces the risk of fluid imbalance, estimated blood loss, and incisional pain; and restores bowel function more quickly [11]. However, despite these potential perioperative benefits, the use of ICUD has been criticized because there is uncertainty on whether it could be performed during surgery without increasing the rate of complications and whether it truly benefits the patient [12].

In this multicenter retrospective study, we analyzed the complications, perioperative outcomes after RARC with extracorporeal urinary diversion (ECUD) or ICUD, and oncologic outcomes in 362 patients with bladder cancer (BC).

\section{MATERIALS AND METHODS}

This retrospective cross-sectional study included $362 \mathrm{pa}-$ tients who underwent RARC with bilateral pelvic lymph node dissection (PLND) at 6 tertiary institutions between 2007 and 2017. A total of 6 surgeons from participating centers performed the procedures, and all of them were experts who had performed $>30$ RARCs. Of them, 4 surgeons performed ECUD only and 2 surgeons performed both

Table 1. Baseline patient characteristics and perioperative parameters

\begin{tabular}{|c|c|c|c|c|}
\hline Baseline characteristic & $\begin{array}{l}\text { Overall population } \\
(n=362)\end{array}$ & $\begin{array}{c}\text { ECUD } \\
(n=278)\end{array}$ & $\begin{array}{c}\text { ICUD } \\
(n=84)\end{array}$ & $\begin{array}{c}\text { p-value } \\
\text { (ECUD vs. ICUD) }\end{array}$ \\
\hline Age $(y)$ & $65.1 \pm 10.6$ & $65.3 \pm 10.5$ & $64.4 \pm 11.0$ & 0.477 \\
\hline Sex & & & & $0.981^{\mathrm{a}}$ \\
\hline Male & $310(85.6)$ & $238(85.6)$ & $72(85.7)$ & \\
\hline Female & $52(14.4)$ & $40(14.4)$ & $12(14.3)$ & \\
\hline Body mass index $\left(\mathrm{kg} / \mathrm{m}^{2}\right)$ & $23.8 \pm 2.9$ & $23.7 \pm 3.0$ & $24.2 \pm 2.3$ & 0.218 \\
\hline Previous abdominal surgery & $46(12.7)$ & $39(14.0)$ & $7(8.3)$ & 0.874 \\
\hline Preoperative chemotherapy & $36(9.9)$ & $27(9.7)$ & $9(10.7)$ & 0.243 \\
\hline Type of urinary diversion & & & & $0.661^{\mathrm{a}}$ \\
\hline lleal conduit & $141(39.0)$ & $110(39.6)$ & $31(36.9)$ & \\
\hline Orthotopic bladder substitution & $221(61.0)$ & $168(60.4)$ & $53(63.1)$ & \\
\hline Type of PLND & & & & $<0.001^{\mathrm{a}, * *}$ \\
\hline Standard PLND & $177(48.9)$ & $167(60.1)$ & $10(11.9)$ & \\
\hline Extended PLND & $185(51.1)$ & $111(39.9)$ & $74(88.1)$ & \\
\hline \multicolumn{5}{|l|}{ Perioperative outcomes } \\
\hline Mean operation time (min) & $523.232 \pm 146.3$ & $510.1 \pm 153.5$ & $566.7 \pm 113.7$ & $0.002^{*}$ \\
\hline Transfusion rate (\%) & 19.9 & 21.2 & 15.5 & 0.248 \\
\hline Mean time to flatus passage $(\mathrm{h})$ & $82.1 \pm 35.3$ & $85.6 \pm 35.6$ & $70.5 \pm 25.3$ & $0.001^{*}$ \\
\hline Mean time to oral intake $(\mathrm{d})$ & $5.1 \pm 2.2$ & $5.3 \pm 2.3$ & $4.4 \pm 1.3$ & $<0.001^{* *}$ \\
\hline Mean time to urinary catheter removal (d) & $16.2 \pm 18.3$ & $16.5 \pm 18.7$ & $15.3 \pm 8.5$ & 0.601 \\
\hline Mean length of hospital stay (d) & $21.0 \pm 12.3$ & $22.4 \pm 12.4$ & $16.6 \pm 8.9$ & $<0.001^{* *}$ \\
\hline
\end{tabular}

Values are presented as mean \pm standard deviation, number only, or number (\%).

ECUD, extracorporeal urinary diversion; ICUD, intracorporeal urinary diversion; PLND, pelvic lymph node dissection. ${ }^{*} p<0.05,{ }^{* *} p<0.001$.

${ }^{a}$ :Pearson chi-square test. 
ICUD and ECUD. The final patient cohort consisted of 278 patients who underwent ECUD and 84 patients who underwent ICUD. This study conformed to the standards of the Declaration of Helsinki and current ethical guidelines, and was approved by the Institutional Review Board of Korea University Anam Hospital (approval number: ED10120). The need for informed consent was waived by the board.

\section{Inclusion and exclusion criteria}

The study participants included patients with muscleinvasive $\mathrm{BC}$ or recurrent high grade non muscle invasive $\mathrm{BC}$ [13]. We excluded patients with stage $\mathrm{cM} 1$ disease, prior extensive abdominal surgery, complicated surgery or any contraindication to the Trendelenburg position. Tumor/ pathologic grade were determined according to the TNM staging system and the 2004 World Health Organization/International Society of Urologic Pathologists classification of papillary urothelial neoplasms, respectively [14]. All research and data collection followed the tenets of the Declaration of Helsinki.

\section{Primary endpoints}

The primary endpoints were the complication rates.
Comparisons were made in accordance with the standardized complications within 90 days of surgery (reported using the Clavien-Dindo classification). The specific complications that were investigated in each complication group were depicted as follows: infectious - urinary tract infection, peritonitis, and fever; respiratory - pneumonia and pulmonary embolism; gastrointestinal_-ileus (mechanical and paralytic), jaundice, and hematochezia; procedure related-transfusion, incisional hernia, bowel injury, and lymphocele; genitourinary-hydronephrosis, uretero-ileal anastomosis site stricture/leakage, and scrotal edema; nervous-obturator injury; vascular-heart failure. In addition, we selected and compared 2 surgeons who could be representative of each group (1 for ECUD and 1 for ICUD). Each surgeon has mastered the learning curve by performing $>100$ RARCs [6]. A total of 75 operations ( 150 cases) were performed by each surgeon during the same period. The types of complications were described in detail, and statistical differences were analyzed.

\section{Secondary endpoints}

The secondary endpoints included perioperative parameters, recurrence patterns, and oncologic outcomes. Perioperative recovery parameters included time to passage of

Table 2. Overall 90-day complications defined according to a modified Clavien system (grade 1-5)

\begin{tabular}{|c|c|c|c|c|c|}
\hline Complication & $\operatorname{ECUD}(n=278 ; n, \%)$ & ICUD (n=84;n, \%) & OR & $95 \% \mathrm{Cl}$ for OR & p-value \\
\hline Overall complications & $171(61.5)$ & $35(41.7)$ & 0.447 & $0.272-0.734$ & $0.001^{*}$ \\
\hline Grade 3-5 complications & $57(20.5)$ & $11(13.1)$ & 0.584 & $0.291-1.174$ & 0.128 \\
\hline Infectious & $43(15.5)$ & $14(16.7)$ & 1.093 & $0.565-2.114$ & 0.791 \\
\hline Grade 3-5 & 6 & 2 & 1.102 & $0.218-5.562$ & 0.907 \\
\hline Respiratory & $2(0.7)$ & $1(1.2)$ & 1.663 & $0.149-18.567$ & 0.676 \\
\hline Grade 3-5 & 0 & 1 & - & - & 0.068 \\
\hline Gastrointestinal & $36(12.9)$ & $4(4.8)$ & 0.336 & $0.116-0.974$ & $0.036^{*}$ \\
\hline Grade 3-5 & 7 & 0 & - & - & 0.142 \\
\hline $\begin{array}{l}\text { Procedure related } \\
\text { (including transfusion) }\end{array}$ & $48(17.3)$ & $8(9.5)$ & 0.504 & $0.228-1.114$ & 0.085 \\
\hline Grade 3-5 & 18 & 4 & 0.722 & $0.238-2.196$ & 0.565 \\
\hline Genitourinary & $26(9.4)$ & $2(2.4)$ & 0.236 & $0.055-1.018$ & $0.036^{*}$ \\
\hline Grade 3-5 & 18 & 1 & 0.174 & $0.023-1.323$ & 0.057 \\
\hline Wound related & $7(2.5)$ & $3(3.6)$ & 1.434 & $0.363-5.672$ & 0.606 \\
\hline Grade 3-5 & 7 & 1 & 0.466 & $0.057-3.846$ & 0.468 \\
\hline Nervous & $2(0.7)$ & $1(1.2)$ & 1.663 & $0.149-18.567$ & 0.676 \\
\hline Grade 3-5 & 0 & 0 & - & - & - \\
\hline Vascular & $3(1.1)$ & $0(0.0)$ & - & - & 0.339 \\
\hline Grade 3-5 & 1 & 0 & - & - & 0.579 \\
\hline Miscellaneous & $4(1.4)$ & $2(2.4)$ & 1.671 & $0.301-9.286$ & 0.553 \\
\hline Grade $3-5$ & 0 & 2 & - & - & $0.010^{*}$ \\
\hline
\end{tabular}

The percentages for the complication category do not sum up because 1 patient can have 2 or more complications.

ECUD, extracorporeal urinary diversion; ICUD, intracorporeal urinary diversion; $n$, patient number; OR, odds ratio; $\mathrm{Cl}$, confidence interval. ${ }^{*} p<0.05$. 
flatus, time to starting oral intake (from liquid diet), time to urinary catheter removal, and length of hospital stay. Then, the recurrence pattern after RARC with ECUD or ICUD was studied. The recurrence type was divided into local and distant, and the specific prevalence of recurrence was deter-

Table 3. Comparison of complication (defined according to a modified Clavien system) rates between ECUD and ICUD performed by 2 expert surgeons (experienced with >100 RARC cases)

\begin{tabular}{cccc}
\hline Complications & $\begin{array}{c}\text { ECUD } \\
(\mathrm{n}=75)\end{array}$ & $\begin{array}{c}\text { ICUD } \\
(\mathrm{n}=75)\end{array}$ & p-value \\
\hline Total complication rate $(\mathrm{n}, \%)$ & $41(54.7)$ & $31(41.3)$ & $0.102^{\mathrm{a}}$ \\
Grades 1 and 2 & $31(41.3)$ & $20(26.7)$ & $0.058^{\mathrm{a}}$ \\
Grades 3-5 & $10(13.3)$ & $11(14.7)$ & $0.814^{\mathrm{a}}$ \\
Infectious & $12(16.0)$ & $11(14.7)$ & $0.821^{\mathrm{a}}$ \\
Grades 3-5 & 0 & 1 & $1.000^{\mathrm{b}}$ \\
Respiratory & $0(0.0)$ & $2(2.7)$ & $0.497^{\mathrm{b}}$ \\
Grades 3-5 & 0 & 1 & $1.000^{\mathrm{b}}$ \\
Gastrointestinal & $10(13.3)$ & $7(9.3)$ & $0.440^{\mathrm{a}}$ \\
Grades 3-5 & 1 & 3 & $0.311^{\mathrm{b}}$ \\
Genitourinary & $12(16.0)$ & $4(5.3)$ & $0.028^{\mathrm{a}, *}$ \\
Grades 3-5 & 3 & 3 & $1.000^{\mathrm{b}}$ \\
Wound related & $3(4.0)$ & $3(4.0)$ & $1.000^{\mathrm{b}}$ \\
Grades 3-5 & 3 & 3 & $1.000^{\mathrm{b}}$ \\
Vascular & $2(2.7)$ & $0(0.0)$ & $0.497^{\mathrm{b}}$ \\
Grades 3-5 & 1 & 0 & $1.000^{\mathrm{b}}$ \\
Nervous & $0(0.0)$ & $2(2.7)$ & $0.497^{\mathrm{b}}$ \\
Grades 3-5 & 0 & 0 & - \\
Miscellaneous & $2(2.7)$ & $2(2.7)$ & $1.000^{\mathrm{b}}$ \\
Grades 3-5 & 2 & 0 & $0.497^{\mathrm{a}}$ \\
\hline Specific complications & & 0 & \\
\hline
\end{tabular}

Specific complications were described in the MATERIALS AND METHODS section.

ECUD, extracorporeal urinary diversion; ICUD, intracorporeal urinary diversion; RARC, robot-assisted radical cystectomy.

${ }^{*} \mathrm{p}<0.05$.

${ }^{a}:$ Pearson chi-square test, ${ }^{b}$ :Fisher exact test.

\section{A}

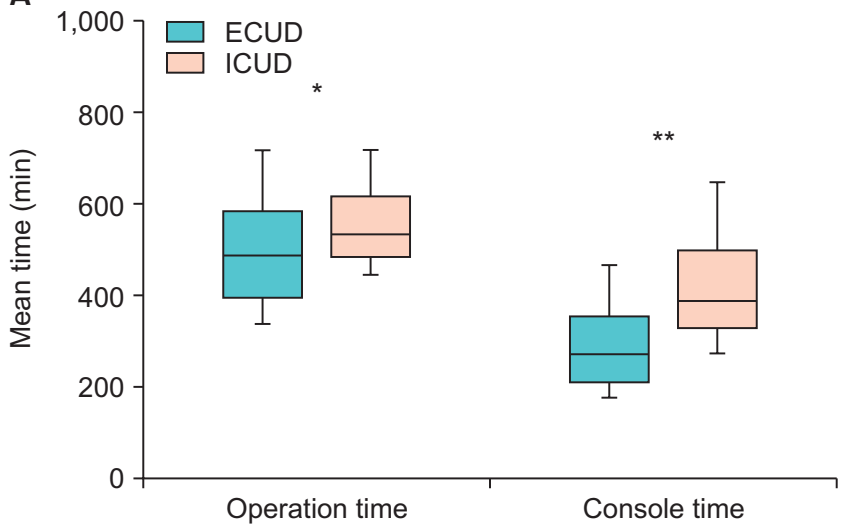

mined. Furthermore, for the analysis of oncologic outcomes, margin positivity rates and 2-year recurrence-free survival (RFS) rates were analyzed. Recurrence was defined as the time from cystectomy to local or metastatic recurrence, based on histologic or radiologic evidence.

\section{Statistical analysis}

Continuous variables were expressed as mean \pm standard deviation, and 1-way analysis of variance was used for the analysis. Additionally, Pearson chi-square test and Fisher exact test were used for analyzing data. The estimated dis-

Table 4. Distribution of recurrence locations among patients with recurrence after RARC with ECUD or ICUD

\begin{tabular}{lrc}
\hline \multicolumn{1}{c}{ Variable } & $\begin{array}{c}\text { ECUD } \\
(\mathbf{n}=\mathbf{2 7 8})\end{array}$ & $\begin{array}{c}\text { ICUD } \\
(\mathbf{n}=84)\end{array}$ \\
\hline Local recurrence & $10(3.6)$ & $2(2.4)$ \\
Cystectomy bed & $4(1.4)$ & $0(0.0)$ \\
PLND template & $6(2.2)$ & $2(2.4)$ \\
Distant recurrence & $25(9.0)$ & $7(8.3)$ \\
Lung & $8(2.9)$ & $1(1.2)$ \\
Liver & $2(0.7)$ & $1(1.2)$ \\
Bone & $5(1.8)$ & $2(2.4)$ \\
Extrapelvic lymph node & $2(0.7)$ & $0(0.0)$ \\
Peritoneal carcinomatosis & $0(0.0)$ & $2(2.4)$ \\
Port site & $2(0.7)$ & $0(0.0)$ \\
Others (brain, adrenal, pancreas, bowel) & $1(0.4)$ & $0(0.0)$ \\
Secondary urothelial carcinoma & & \\
Upper urinary tract & $2(0.7)$ & $0(0.0)$ \\
Urethra & $1(0.4)$ & $0(0.0)$ \\
Multiple recurrence & $2(0.7)$ & $1(1.2)$ \\
\hline
\end{tabular}

Values are presented as number of recurrence sites (\%).

RARC, robot-assisted radical cystectomy; ECUD, extracorporeal urinary diversion; ICUD, intracorporeal urinary diversion; PLND, pelvic lymph node dissection.

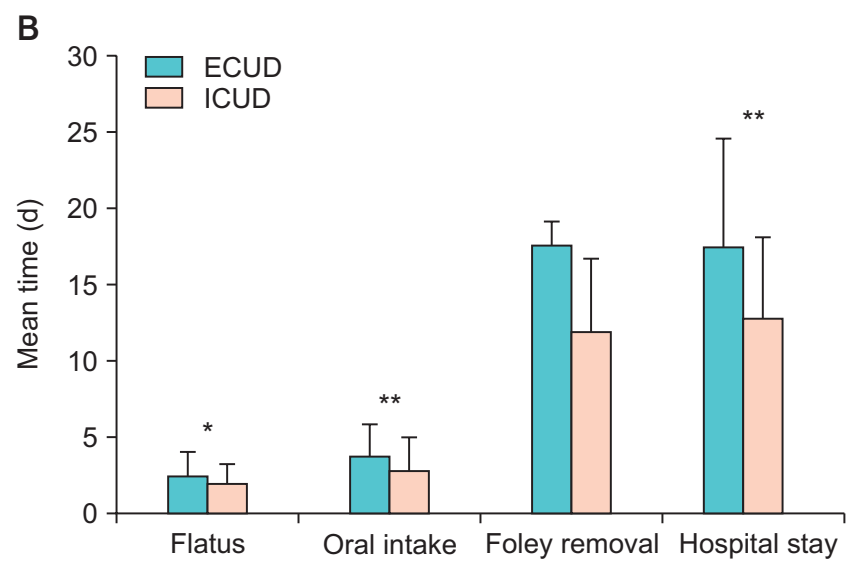

Fig. 1. Comparison of perioperative valuables between the extracorporeal urinary diversion (ECUD) and intracorporeal urinary diversion (ICUD) groups. (A) Total operation time and console time and (B) recovery parameters (time to flatus, time to oral intake, time to urinary catheter removal, and length of hospital stay). ${ }^{*} p<0.05,{ }^{* *} p<0.001$. 
Table 5. Oncologic outcomes

\begin{tabular}{lccc}
\hline \multicolumn{1}{c}{ Pathologic outcomes } & $\begin{array}{c}\text { ECUD } \\
(\mathbf{n}=\mathbf{2 7 8})\end{array}$ & $\begin{array}{c}\text { ICUD } \\
(\mathbf{n}=84)\end{array}$ & p-value \\
\hline Histologic type & & & \\
$\quad$ Urothelial carcinoma & $278(100.0)$ & $84(100.0)$ & 1.000 \\
Pathologic tumor stage & & & $0.152^{\mathrm{a}}$ \\
T0/Ta/Tis & $38(13.7)$ & $18(21.4)$ & \\
T1/T2 & $153(55.0)$ & $38(45.2)$ & \\
T3/T4 & $87(31.3)$ & $28(33.3)$ & \\
Soft tissue margin positive & $7(2.5)$ & $2(2.4)$ & 0.944 \\
Lymph node yield & $15.1 \pm 12.1$ & $29.8 \pm 13.6$ & $<0.001^{*}$ \\
Pathologic nodal stage & & & $0.116^{\mathrm{a}}$ \\
$\quad$ Nx/N0 & $227(81.7)$ & $62(73.8)$ & \\
$\quad$ N1/N2/N3 & $51(18.3)$ & $22(26.2)$ & \\
Recurrence-free survival & 83.2 & 88.6 & $0.496^{\mathrm{b}}$ \\
for 2 years & & & \\
\hline
\end{tabular}

Values are presented as number (\%), mean \pm standard deviation, or a percentage only.

ECUD, extracorporeal urinary diversion; ICUD, intracorporeal urinary diversion; SD, standard deviation.

${ }^{*} \mathrm{p}<0.001$.

${ }^{\mathrm{a}}$ :Pearson chi-square test, ${ }^{\mathrm{b}}$ :log-rank test.

tribution of RFS was obtained using Kaplan-Meier analysis, and the log-rank test was used to compare the Kaplan-Meier curves between the groups. All analyses were performed using SPSS version 20.0 (SPSS Inc, Chicago, IL, USA). Results were considered statistically significant if the p-value was less than 0.05 .

\section{RESULTS}

The mean duration of follow-up was $34.7 \pm 25.5$ months. We observed no difference in age, sex, body mass index, incidence of previous abdominal surgery, incidence of neoadjuvant chemotherapy, and type of UD (ileal conduit or orthotopic neobladder substitution) between the 2 groups. In the ICUD group, the rate of performing extended PLND was significantly higher than that of standard PLND $(p<0.001)$ (Table 1).

\section{Complications I}

In the ECUD group, 171 patients (61.5\%) experienced at least 1 complication of any grade within 90 days of surgery, whereas there were 35 such patients (41.7\%) in the ICUD group $(\mathrm{p}=0.001)$. In the analysis of overall complication and major complication rates (grade $3-5$ ), there were no significant differences between the 2 groups. The incidence of gastrointestinal complications in the ICUD group showed a decreasing trend $(\mathrm{p}=0.036)$, and there were no major complications in the ICUD group. The incidence of genitourinary complications was also lower $(\mathrm{p}=0.036)$ in the ICUD group (Table 2). In the analysis of complication rates in the genitourinary tract, there were significant differences between the 2 groups $(p=0.036)$. In terms of the type of complication, hydronephrosis (20 of 28) was the most common, followed by acute kidney injury ( 5 of 28), urinary stone (2 of 28 ), and bladder neck stricture (1 of 28 ).

\section{Complications II}

We performed a head-to-head comparison between 2 expert surgeons from each group. The total complication and major complication rates showed no difference, whereas the minor complication rates showed a decreasing trend in the ICUD group (ECUD vs. ICUD: 31 [41.3\%] vs. 20 [26.7\%], $\mathrm{p}=0.058$ ). The urine leakage rates were significantly lower (ECUD vs. ICUD: 9 [12.0\%] vs. 0 [0.0\%, $p=0.002$ ) in the ICUD group (Table 3).

\section{Perioperative parameters}

The ICUD group had significantly longer total operation time and console time, whereas the postoperative transfusion rate showed no difference ( $21.2 \%$ vs. $15.5 \%$, $\mathrm{p}=0.248)$. Recovery parameters, such as mean time to passage of flatus, start of oral intake, and length of hospital stay, were significantly shorter in the ICUD group than in the ECUD group ( $p=0.001$, $\mathrm{p}<0.001$, and $\mathrm{p}<0.001$, respectively), except for the mean time to Foley catheter removal $(\mathrm{p}=0.601)$ (Table 1, Fig. 1).

\section{Recurrence patterns}

Local/distant recurrence occurred in $10(3.6 \%)$ and 25 (9.0\%) patients in the ECUD group and in 2 (2.4\%) and 7 (8.3\%) patients in the ICUD group, respectively. Concerning both types of recurrence, there were no significant differences in the recurrence pattern between the 2 groups. Only 3 patients experienced recurrence of secondary urothelial carcinoma in the upper urinary tract (2 patients) and urethra (1 patient). The diversion of recurrence locations is shown in Table 4.

\section{Oncologic outcomes}

There was no difference in the margin positivity rate $(p=0.944)$. In pathologic results, there were no differences between the 2 groups in numbers of lymph-node-positive patients $(\mathrm{p}=0.523)$, lymph node density $(\mathrm{p}=0.127)$, and pathologic tumor stage or nodal stage $(p=0.152, p=0.116)$. The lymph node yield was significantly higher in the ICUD group than in the ECUD group (29.8 \pm 13.6 vs. $15.1 \pm 121, \mathrm{p}<0.001)$. The 2-year RFS showed no significant difference between the 2 groups (ECUD vs. ICUD: $83.2 \%$ vs. $88.6 \%$, $\mathrm{p}=0.496$ ) (Table 5). 


\section{DISCUSSION}

The aim of our study was to determine whether ICUD has clinical benefits compared with ECUD. Our results suggest that the ICUD approach is beneficial in terms of lower complication rates and better perioperative outcomes, as indicated by recovery parameters, while maintaining similar oncologic outcomes to those of ECUD.

Our study showed a significant advantage of ICUD over ECUD for patients with regard to overall complication rates and gastrointestinal-related complications. The International Robotic Cystectomy Consortium conducted a comparative analysis of ICUD and ECUD. Overall, a trend favoring ICUD over ECUD was noted ( $41 \%$ vs. $49 \%$, $p=0.05$ ). Gastrointestinal complications were significantly lower in the ICUD group ( $p<0.001)$. Additionally, Bochner et al. [12] reported that the most common complications during cystectomy were gastrointestinal-, infection-, and wound-related problems in their prospective study. Complications related to bowel resection are undoubtedly one of the biggest problems faced by urologists and an important reason for prolonged hospital stays. In this regard, the exposure time of the peritoneum to external air was proportional to intestinal inflammation and oxidative stress response in experimental models using white rats. This leads to intestinal paralysis or intestinal obstruction and delayed restoration of bowel function [15]. Other studies comparing laparoscopy and laparotomy in bowel surgery have shown a decrease in the levels of cytokines, including interleukin (IL)-1, $\mathbb{L}-6$, and tumor necrosis factoralpha, and a reduction in systemic immunosuppression in endoscopic surgery as compared with open surgery $[15,16]$.

In our study, the ICUD group showed a decreasing trend of genitourinary complications, which consisted mostly of urinary leakage or anastomosis site stricture. Furthermore, as shown in Table 5, in the analysis of the surgeons who overcame the learning curve, lower minor complication rates were found in the ICUD group in terms of ureteral complications, whereas the major complication rates were similar. ECUD requires more ureteral mobilization and longer ureteral length, which can result in ureteral redundancy, kinking, and devascularization [10]. On the other hand, in the ICUD approach, anastomoses are made at the position, resulting in less ureter mobilization and tension. In several large ICUD series, a low stricture rate of approximately $3 \%$ was reported [17,18].

Considering the potential advantages of ICUD, patients undergoing $\mathrm{RC}$ are often older and have several comorbidities, which are risk factors for increased complications [19]. There is evidence that RARC may have an advantage in reducing the surgical stress response compared with open surgery in older patients or other vulnerable patients [20,21]. Reduction in the stress response can be achieved through minimal-access surgery [22]. These concepts are in line with the benefits of ICUD, such as minimizing the handling time of organs in the abdominal cavity, as well as decreasing bowel manipulation/exposure and reducing insensible fluid losses $[23,24]$. Our results showed a relatively shorter length of stay (days) in patients who underwent ICUD than in those who underwent ECUD (16.6 \pm 8.9 vs. $22.4 \pm 12.4$, p $<0.001$ ), as well as shorter time to passage of flatus and time to starting oral intake. Improvements in recovery parameters are especially important in elderly patients with comorbidities who undergo RARC. There is increasing evidence showing that patients who are susceptible to the morbidity of major surgery may be the ones who will benefit from a robotic approach [20].

In the analysis of recurrence patterns, the overall rates of local and distant recurrence did not show any difference between the 2 groups. With respect to incidence of distant recurrence of solid organs, lung, bone and liver remain the most frequent sites and homologous to previous studies [25]. The incidence rates of peritoneal carcinomatosis and port site recurrence were not significantly different between the 2 groups. Although no statistically significant differences were found, more attention is needed to avoid urine leakage when performing the procedure (after removing the specimen and transferring it into the endopouch). This is important because the follow-up strategy should reflect the risk of extravesical recurrence [26], and this type of recurrence was observed in the ECUD group only.

Despite several potential perioperative benefits, the use of RARC has been criticized owing to the paucity of reports about a few oncologic concerns. According to the data available to date, RARC can implement adequate PLND in terms of its territory and yield, and has an acceptable positive surgical margin (PSM) rate. In addition, it is possible to avoid urine leakage or inadvertent bladder injury and safely perform the surgery while adhering to the oncologic principles. Furthermore, it showed similar results to those of an open series in patients with mostly T3 or higher stages. In our study, the PSM ( $\mathrm{p}=0.944)$ and lymph-node-related factors did not differ, except that the lymph node yield and shortterm RFS $(p=0.496)$ did not show inferior results to those of ECUD. Although ICUD remains a complex procedure, the outcomes of this study agree with those of previous studies, thereby demonstrating the potential benefits of ICUD after RARC. This will contribute to ICUD gaining popularity as a viable alternative to ECUD. Theoretically, there is no difference in the oncologic outcome between ECUD and 
ICUD. Nevertheless, the reason why we evaluated the oncologic outcome is that there is a slight procedural difference between the 2 methods after a cystectomy. In the case of ICUD, the time during which the specimen stays inside the body is inevitably longer, according to the surgical principle. Accordingly, precaution is needed because of the possibilities of local tumor spillage and urine leakage even after removing the specimen.

There are some limitations in this study. First, the medical records of consecutive patients were retrospectively reviewed. The number and timing of operations performed in each surgeon are different, and this may have affected the outcome of our study. Therefore, this study is limited by biases such as lack of randomization, patient selection bias. Better designed research would be needed in the future.

\section{CONCLUSIONS}

In this multicenter trial, there were decreasing trends in overall, gastrointestinal, and genitourinary complications in the ICUD group. The ICUD group showed significantly improved recovery parameters compared with the ECUD group. Analysis of oncologic outcomes at intermediate-term follow-up revealed no differences in the PSM rate, recurrence pattern, or RFS between the 2 UD techniques. In the future, the results of prospective, randomized controlled trials between RARC and open RC are awaited to confirm the current findings.

\section{CONFLICTS OF INTEREST}

The authors have nothing to disclose.

\section{ACKNOWLEDGMENTS}

This research was supported by a grant of Korea University Medical Center and Korea University Anam Hospital, Seoul, Korea and by a Korean Endourological Society Grant.

\section{AUTHORS' CONTRIBUTIONS}

Research conception and design: Ji Sung Shim and Seok Ho Kang. Data acquisition: Tae Gyun Kwon, Koon Ho Rha, Young Goo Lee, Ji Youl Lee, and Byong Chang Jeong. Statistical analysis: Jong Hyun Pyun. Data analysis and interpretation: Sung Gu Kang. Drafting of the manuscript: Ji Sung Shim. Critical revision of the manuscript: Seok Ho Kang. Approval of the final manuscript: all authors.

\section{REFERENCES}

1. Yuh B, Wilson T, Bochner B, Chan K, Palou J, Stenzl A, et al. Systematic review and cumulative analysis of oncologic and functional outcomes after robot-assisted radical cystectomy. Eur Urol 2015;67:402-22.

2. Leow JJ, Reese SW, Jiang W, Lipsitz SR, Bellmunt J, Trinh QD, et al. Propensity-matched comparison of morbidity and costs of open and robot-assisted radical cystectomies: a contemporary population-based analysis in the United States. Eur Urol 2014;66:569-76.

3. Hussein AA, May PR, Jing Z, Ahmed YE, Wijburg CJ, Canda $\mathrm{AE}$, et al. Outcomes of intracorporeal urinary diversion after robot-assisted radical cystectomy: results from the International Robotic Cystectomy Consortium. J Urol 2018;199:1302-11.

4. Challacombe BJ, Bochner BH, Dasgupta P, Gill I, Guru K, Herr $\mathrm{H}$, et al. The role of laparoscopic and robotic cystectomy in the management of muscle-invasive bladder cancer with special emphasis on cancer control and complications. Eur Urol 2011;60:767-75.

5. Styn NR, Montgomery JS, Wood DP, Hafez KS, Lee CT, Tallman C, et al. Matched comparison of robotic-assisted and open radical cystectomy. Urology 2012;79:1303-8.

6. Collins JW, Tyritzis S, Nyberg T, Schumacher MC, Laurin O, Adding C, et al. Robot-assisted radical cystectomy (RARC) with intracorporeal neobladder - what is the effect of the learning curve on outcomes? BJU Int 2014;113:100-7.

7. Collins JW, Wiklund PN, Desai MM, Goh AC, Gill IS. Total intracorporeal robotic cystectomy: are we there yet? Curr Opin Urol 2013;23:135-40.

8. Desai MM, de Abreu AL, Goh AC, Fairey A, Berger A, Leslie $S$, et al. Robotic intracorporeal urinary diversion: technical details to improve time efficiency. J Endourol 2014;28:1320-7.

9. Patel HR, Santos PB, de Oliveira MC, Müller S. Is roboticassisted radical cystectomy (RARC) with intracorporeal diversion becoming the new gold standard of care? World J Urol 2016;34:25-32.

10. Dason S, Goh AC. Contemporary techniques and outcomes of robotic cystectomy and intracorporeal urinary diversions. Curr Opin Urol 2018;28:115-22.

11. Wilson TG, Guru K, Rosen RC, Wiklund P, Annerstedt M, Bochner $\mathrm{BH}$, et al. Best practices in robot-assisted radical cystectomy and urinary reconstruction: recommendations of the Pasadena Consensus Panel. Eur Urol 2015;67:363-75.

12. Bochner BH, Dalbagni G, Sjoberg DD, Silberstein J, Keren Paz GE, Donat SM, et al. Comparing open radical cystectomy and robot-assisted laparoscopic radical cystectomy: a randomized clinical trial. Eur Urol 2015;67:1042-50.

13. van den Bosch S, Alfred Witjes J. Long-term cancer-specific 
survival in patients with high-risk, non-muscle-invasive bladder cancer and tumour progression: a systematic review. Eur Urol 2011;60:493-500.

14. Montironi R, Lopez-Beltran A. The 2004 WHO classification of bladder tumors: a summary and commentary. Int J Surg Pathol 2005;13:143-53.

15. Lee SW, Feingold DL, Carter JJ, Zhai C, Stapleton G, Gleason $\mathrm{N}$, et al. Peritoneal macrophage and blood monocyte functions after open and laparoscopic-assisted cecectomy in rats. Surg Endosc 2003;17:1996-2002.

16. Sammour T, Kahokehr A, Chan S, Booth RJ, Hill AG. The humoral response after laparoscopic versus open colorectal surgery: a meta-analysis. J Surg Res 2010;164:28-37.

17. Desai MM, Gill IS, de Castro Abreu AL, Hosseini A, Nyberg $\mathrm{T}$, Adding $\mathrm{C}$, et al. Robotic intracorporeal orthotopic neobladder during radical cystectomy in 132 patients. J Urol 2014;192:1734-40.

18. Tan WS, Lamb BW, Tan MY, Ahmad I, Sridhar A, Nathan S, et al. In-depth critical analysis of complications following robotassisted radical cystectomy with intracorporeal urinary diversion. Eur Urol Focus 2017;3:273-9.

19. Froehner M, Brausi MA, Herr HW, Muto G, Studer UE. Complications following radical cystectomy for bladder cancer in the elderly. Eur Urol 2009;56:443-54.

20. Knox ML, El-Galley R, Busby JE. Robotic versus open radical cystectomy: identification of patients who benefit from the robotic approach. J Endourol 2013;27:40-4.

21. Richards KA, Kader AK, Otto R, Pettus JA, Smith JJ 3rd, Hemal AK. Is robot-assisted radical cystectomy justified in the elderly? A comparison of robotic versus open radical cystectomy for bladder cancer in elderly $\geq 75$ years old. J Endourol 2012;26:1301-6.

22. Ost MC, Tan BJ, Lee BR. Urological laparoscopy: basic physiological considerations and immunological consequences. J Urol 2005;174:1183-8.

23. Goh AC, Gill IS, Lee DJ, de Castro Abreu AL, Fairey AS, Leslie $S$, et al. Robotic intracorporeal orthotopic ileal neobladder: replicating open surgical principles. Eur Urol 2012;62:891-901.

24. Jonsson MN, Adding LC, Hosseini A, Schumacher MC, Volz D, Nilsson A, et al. Robot-assisted radical cystectomy with intracorporeal urinary diversion in patients with transitional cell carcinoma of the bladder. Eur Urol 2011;60:1066-73.

25. Yafi FA, Aprikian AG, Fradet Y, Chin JL, Izawa J, Rendon R, et al. Surveillance guidelines based on recurrence patterns after radical cystectomy for bladder cancer: the Canadian Bladder Cancer Network experience. BJU Int 2012;110:1317-23.

26. Babjuk M, Burger M, Zigeuner R, Shariat SF, van Rhijn BW, Compérat E, et al. EAU guidelines on non-muscle-invasive urothelial carcinoma of the bladder: update 2013. Eur Urol 2013;64:639-53. 